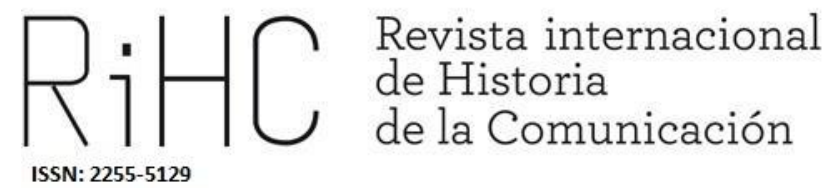

\title{
PROSOPOGRAFÍA Y CAREER RESEARCH EN LA HISTORIA DEL PERIODISMO (Y UNA PROPUESTA DE APLICACIÓN)
}

\section{Prosopography and career research in journalism history (and a proposal for application)}

DOI: http://dx.doi.org/10.12795/RiHC.2018.i11.06

Recibido: 20/9/2018

Aceptado: 16/10/2018

Publicado: $15 / 12 / 2018$

Javier Díaz Noci ORCID (D) https://orcid.org/0000-0001-9559-4283

Universitat Pompeu Fabra (Barcelona), javier.diaz@upf.edu

Resumen: Esta es una propuesta de tipo metodológico. Se propone la aplicación de una técnica bien conocida en la historiografía contemporánea para grupos sociales y profesionales, la prosopografía o biografía colectiva, en este caso desplazando el tradicional centro de atención dispensados a las organizaciones de medios de comunicación como objeto principal de estudio hacia la historia social de las personas que participan en dichas estructuras, y principalmente a los periodistas. Proponemos complementar la aplicación de la prosopografía, y el diseño, empleo y explotación de 
bases de datos ad hoc, con otras metodologías propias de las ciencias sociales, como las entrevistas semiestructuradas, y focalizadas en función de las categorías y campos empleados en la prosopografía, e incluso el análisis de redes sociales, con objeto de sentar las bases de una investigación sobre carreras profesionales. Se plantea la aplicación de estas técnicas al estudio de la historia de los medios digitales.

Palabras clave: Historiografía, Prosopografía, Entrevistas semiestructuradas, Análisis de redes sociales, Etnografía de las redacciones.

\begin{abstract}
This is a methodological proposal. In this article we propose the application of a well-known technique in contemporary historiography for career research of specific social and professional groups, prosopography or systematic collective biography. In doing so, we propose to displace the attention traditionally focused on media organizations towards a social history of the people involved in news reporting and production, namely journalists. We propose to complete prosopography and the design, use and exploitation of ad hoc databases combined with the application of some other widely used techniques of the social sciences, semi-structured, focused interviews (around categories and fields used in the aforementioned prosopography) and even social media networks analysis. It is highlighted the application of such methods to online media and digital journalists' career research.
\end{abstract}

Keywords: Historiography, Prosopography, Semi-structured interviews, Social network analysis, Newsroom ethnography.

\title{
Introducción y estado de la cuestión
}

Los métodos biográficos aplicados a la historia de la comunicación (sobre las características propias de esta disciplina en concreto, véase, por ejemplo, Pooley y Park, 2013, y Simonson y Park, 2015) y a la historia del periodismo se han practicado, desde diversos enfoques metodológicos, desde el inicio mismo de la historiografía sobre este fenómeno social. Han adquirido, además, una renovada importancia en los últimos años, desde luego en propuestas como las de Pont-Sorribes, Luis y Sanmartí, 2017, por un lado, y Soriano, 2017, por otro, ambas publicadas en el número 22(1) de la revista especializada Historia de la Comunicación Social. El primero de esos artículos, además, subraya desde el principio la pertinencia de unir la historiografía como campo y técnica científica con una aproximación, si se quiere, más próxima a las ciencias sociales que a las humanas, si es que esa división planteada en sentido estricto resulta operativa para los objetivos de nuestras investigaciones. En concreto, dichos autores planteaban la conveniencia de plantearse una etnografía de las redacciones (newsroom ethnography). Si dichos investigadores se han basado, para sus investigaciones sobre la Transición democrática española, sobre todo en la técnica del 
focus group (Pont-Sorribes, Luis y Sanmartí, 2017: 144), nosotros propondremos emplear también, y sobre todo, las entrevistas semiestructuradas, tal y como hacemos en Díaz Noci, 2018. En aquel texto, redactado a partir de una comunicación sobre los orígenes y primeros años de consolidación de sendos medios digitales en la Barcelona post-olímpica (el Diari de Barcelona digital y La Malla, dos medios innovadores de iniciativa pública) que presentamos al XV Congreso de Historia de Barcelona en octubre de 2017, planteábamos algunas cuestiones sobre historiografía de medios digitales (sobre la periodización de esta forma de periodismo, véase nuestra propuesta en Díaz Noci, 2013) y, sobre todo, sobre la profesión periodística en dichos medios y en ese entorno, el digital, que plantea algunos retos a la historiografía (véase Brúgger y Kolstrup, 2002, Brügger, 2010 y 2012, y Weller, 2013), que pretendemos recoger y ampliar ahora aquí. En concreto, lo que propondremos es aplicar los métodos de la prosopografía histórica en forma de biografías colectivas (véase, por ejemplo, KeatsRohan, 2007; Oldfield y Day, 2011), dé eso luego paso a la producción de diccionarios biográficos (impresos o, más dinámicos, en línea) como los que son habituales para otros grupos sociales, por ejemplo parlamentarios o incluso docentes universitarios (Corell Domenech, 2013, Meyen y Wiedemann, 2017), o para periodistas de otras épocas, como el Dictionnaire des Journalistes, 1600-1789 dirigido por Jean Sgard (Sgard, 1991, en su edición impresa, o la página web del proyecto, renovada a lo largo de $\operatorname{los}$ años $\left.{ }^{1}\right)$.

La combinación de ambas metodologías, técnicas y aproximaciones (humanidades y ciencias sociales, historiografía y etnografía, entrevistas y prosopografía) tienen como objetivo ir más allá de las biografías individuales de periodistas destacados (Soriano, 2017) para proponer una biografía colectiva de un grupo social y una investigación sobre la profesión (o la carrera) de informador social, lo que se denomina una career research (Cohen y Mallon, 2001; Tsui y Lee, 2012). La posibilidad de disponer de un grupo humano y social cuyos componentes están vivos y activos, como las primeras redacciones digitales, nos permite, por un lado, investigar sobre momentos de cambio social -el paso de una determinada manera de informar a otra, en un periodo de transición determinado por la emergencia y consolidación de la tecnología digital en línea-, preguntarnos por desarrollos longitudinales más que pretender hacer una foto fija de un determinado momento social; y por otro, nos permite aplicar técnicas que, para otro momento histórico más distante, resultarían imposibles. Por lo tanto, en nuestra propuesta la triangulación metodológica resultará un planteamiento irrenunciable de origen.

La propuesta que a continuación desarrollamos no desdeña enunciar cuál es nuestro punto de vista interpretativo: la historia social, entendida no sólo pero sí sobre todo como una history from below, como lo han planteado historiadores como Eric

\footnotetext{
${ }^{1}$ http://dictionnaire-journaux.gazettes18e.fr/
} 
Hobsbawm y otros, al tratar el periodismo como una actividad social más que exclusivamente una iniciativa empresarial, y por tanto poniendo el acento en la relevancia de personas y grupos sociales. Nos interesa, naturalmente, el papel de empresas e instituciones en las iniciativas mediáticas, pero más que nada nos importa conocer cuál es el papel de los profesionales de la información en el rumbo que la comunicación, en este caso periodística, adopta condicionada por determinadas cuestiones, como la aparición e implementación de nuevas tecnologías, por entender que se trata de elementos constitutivos de las sociedades que estudiamos (Juliá, 1989: 11, Samuel, 1985), cuya importancia debe ser estudiada tanto de modo sincrónico como diacrónico. Pretendemos explícitamente ir más allá de la historia de la prensa, o de los medios (véase, por ejemplo, Raymond, 1999, Broersma, 2011, y para el caso concreto del periodismo digital, Scott, 2005).

\section{Objeto de estudio y método}

La biografía como género científico y, concretamente, historiográfico, se diferencia de otro tipo de biografías porque, en primer lugar, entiende que explicando la trayectoria vital y, sobre todo, profesional y pública de los miembros de determinados colectivos ayuda a explicar las razones de los comportamientos de los grupos sociales (para el caso de los periodistas, y desde una perspectiva histórica, veáse por ejemplo Corell Domenech, 2013, Oldfield, 2015, Cruz y López-Cordón, 2016 y Onandia Martínez, 2018). En este sentido, nos acogemos a las palabras de uno de los pioneros de esta técnica historiográfica, J. E. Neale, cuando en 1951 aseguraba que no era posible entender la naturaleza y funcionamiento de un grupo social sin conocer los detalles de los individuos que lo componen. Para que sean realmente científicas, las biografías colectivas de determinados colectivos sociales deben reunir, al menos, un requisito: confeccionarse a partir de material de primera mano, bien sea documentación primaria bien sea, para grupos sociales como el de nuestro caso, mediante entrevistas semiestructuradas u otro tipo de técnicas grupales, como los paneles de expertos o métodos Delphi (lo que se denomina métodos de consenso).

De alguna manera, esta propuesta entronca con la sociologçia de las redacciones que fue popularizada en los últimos años de la década de 1970 por Gaye Tuchman (Tuchman, 1978), y que fue recogida en España, aunque se trate de un estudio más bien téorico que no basado en la recogida de datos empíricos y de un estudio de campo propiamente dicho, por Miquel Rodrigo Alsina, bebiendo a su vez de un campo que para nuestro objeto y nuestro enfoque no resulta pertinente, como era la semiótica (o sociosemiótica) entonces tan en boga en las facultades de comunicación españolas y latinoamericanas (véase Rodrigo, 1989). En realidad, tanto estos trabajos, 
que se centraban en aspectos importantes como los mecanismos (también profesionales) de construcción de la noticia desde un punto de vista sociológico, el primero, y más bien semiótico, el segundo, como otros anteriores, llevados a cabo en las décadas anteriores en los Estados Unidos desde un punto de vista más de la profesión periodística que puramente académico (nos referimos a Epstein, 1973, y Gans, 1979) para luego pasar a centrarse en estudios sobre televisión (Lull, 1988), incorporando estudios de audiencias (Lull, 1990), y una perspectiva propiamente etnográfica, cuya piedra angular metodológica se halla en Savill-Troike, 1989 (para algunas aproximaciones etnográficas a los medios y su consumo, de nuevo, Lull, 1990, y posteriormente y ya enfrentándose a los particulares problemas de la revolución digital, Paterson y Domingo, 2008, y Usher, 2014 y 2015, por sólo citar dos ejemplos), que se añadían así a las perspectivas más puramnete histórica y sociológica, que a veces se aunaban en un solo investigador (Schudson, 1981 y 1990, por ejemplo). Sin embargo, la mayoría de estos estudios, salvo los de Michael Schudson, dan una importancia marginal a la perspectiva historiográfica. Nuestra propuesta concreta será, por tanto, incorporar técnicas propias de la historiografía al estudio de las redacciones informativas

Si explicamos la trayectoria vital de, por ejemplo, las redacciones de unos casos representativos (como entendemos que son los medios apuntados) y lo relacionamos también con aspectos de estructura empresarial, decisiones políticas y consumo de medios e información, podremos obtener explicaciones profundas (thick descriptions) de determinados fenómenos sociales, como el paso del sistema mediático tal como lo conocíamos hasta la década de 1990 a un sistema digital. La triangulación metodológica nos ayuda ciertamente, como explicaremos en la siguiente sección.

Por supuesto, la revisión documental es otra técnica nada desdeñable. Además de un análisis de contenido, método que no vamos a desarrollar aquí, de los propios medios, todo documento que nos pueda ayudar a trazar el contexto en que trabajan los grupos sociales objeto de nuestro estudios una información preciosa. Por ejemplo, y para el caso que nos ayuda a ilustrar nuestro punto de vista: el Grup de Periodistes Digitals produjo, durante la época que estudiamos (finales de la década de 1990 y primera década del nuevo siglo) algunos informes que destacan por ser de los primeros que se llevaron a cabo y que sin duda reforzaron la conciencia de pertenecer a un colectivo en formación: véase, así, el Informe sobre la situació laboral i professional del periodista digital a Catalunya (Asensio et al. 2003) y algunas conclusiones Calvo, 2005. 


\section{Prosopografía y periodistas}

En todo caso, y volviendo a las biografías colectivas o prosopografías, se confeccionarán siguiendo las mismas pautas, como ya han hecho, para diferentes colectivos sociales, investigadores como E. Bunout (Bunout, 2016). Es más: planteamos la confección de bases de datos, como ya se ha hecho, por ejemplo, en Onandia Martínez, 2018, de manera que los datos se estructuran como campos de diverso tipo (del texto libre a los teasuros documentales), de manera que después los resultados puedan ofrecerse en forma de tablas y estadísticas, cuando proceda, lo cual permitirá no sólo obtener conclusiones para cada uno de los individuos biografiados, sino sobre también y sobre todo sobre las instituciones a las que pertenecen (o pertenecieron) y que de alguna manera representan. Eso, sin duda, redundará en beneficio de la historia de las propias instituciones que, como se ha dicho, son sin duda importantes, aunque no los únicos actores del fenómeno social estudiado, historia que formará parte de la thick description que se pretende confeccionar con esta propuesta.

En esta propuesta planteamos que uno de los resultados previstos sea un diccionario biográfico de grupo. Los diccionarios de grupo son muy conocidos en el mundo académico anglosajón. Los Companion pertenecen a esta modalidad. La producción de diccionarios de periodistas, con criterios científicos o no, ha sido más bien escasa. En España, concretamente, existen algunos intentos que sí conviene destacar. Durante el siglo XIX se llegaron a publicar algunos libros de este tipo. Además de algunas obras menores (Galería en miniatura de los más célebres periodistas, folletistas y articulistas de Madrid y su Apéndice, de Manuel Eduardo Gorostiza y Cepeda, 1822; Galería de la prensa o colección de retratos políticos de los periodistas de España, hechos al daguerrotipo, apenas cuatro semblanzas de otros tantos periodistas escritas por Juan Pérez Calvo, 1846), hubo un primer intento en 1875: el Catálogo de ilustres periodistas españoles desde el siglo XVII (1600-1875), de Juan Pérez de Guzmán y Gallo. "Se trata", indica José Altabella (prólogo a López de Zuazo, 1980: 4), "de una simple enumeración alfabética de nombres, seguidos -en algunos casos- de la ciudad de donde son naturales".

Sin embargo, el primer intento de hacer un diccionario biográfico sistemático de los periodistas españoles de la época es el Ensayo de un catálogo de periodistas españoles del siglo XIX, de Manuel Osorio y Bernard, publicado en 1904 (existe un facsímil dado a la luz pública un siglo después, en 2004), un volumen de algo más de 500 páginas de apretada letra (primero se publicó, entre 1903 y 1904, en forma de cuadernillos), en el que se recogen, por orden alfabético, el nombre y principales datos de unos 10.000 periodistas: nombre y apellidos, y una breve reseña de apenas unas líneas que a menudo se reduce a su principal actividad (redactor o director en tal o cual diario o revista) y a veces se extiende algo más, dando cuenta del lugar y fecha de nacimiento, estudios, cargos y puestos de trabajo. Se trata, en palabras de su autor, de una "ligera enumeración de cuantos han consagrado sus esfuerzos a la labor periodísticas, con 
somera indicación del servicio prestado y lugares y fechas en que lo ha sido", que no oculta -recordemos que fue la obra de una sola persona- sus "grandísimas deficiencias", una de las cuales radicaba precisamente en uno de los principales requisitos para que un empeño de estas característica pueda ser considerado realmente científico: "No son muchas", decía Osorio, "ni muy dignas de crédito las fuentes de información de que me he servido". No hay que olvidar, y el propio autor lo tiene bien en cuenta, en qué momento se produce esta obra: justo cuando se está comenzando a reivindicar un cierto estatus para el periodismo, "necesidad social y género literario" (Osorio, 1904: viii-x. Ochenta años después se publica otro diccionario similar en su concepción y desarrollo sobre periodistas españoles del siglo xx, y que de hecho pretende ser la continuación de aquel. Es el Catálogo de periodistas españoles del siglo Xx, del profesor de la Universidad Complutense de Madrid Antonio López de Zuazo Melgar. Se trata de un total de 14.325 fichas confeccionadas entre 1976 y 1980 , con una breve descripción en cada uno de los autores de los puestos de trabajo y cargos desempeñados como periodistas, e indicación del lugar y fecha de nacimiento. El libro constituye un catálogo que pretende ir más allá de la mera acumulación de datos, y ser el primer paso para futuras entregas. De hecho, el 1990 el profesor López de Zuazo lee en la Universidad Complutense su tesis doctoral, Bases metodológicas para la catalogación de periodistas españoles. Pretende basarse, entre otras fuentes, en la lista de miembros de las asociaciones de periodistas, en el Registro Oficial de Periodistas de la época franquista (fue creado en 1938) y en otras asociaciones profesionales. A pesar de ser un trabajo inconcluso (el esfuerzo es ciclópeo para una sola persona) y de constituir más bien una "foto fija" del censo aproximado de periodistas españoles en activo entre 1976 y 1980 (aunque incluye la ficha de unos 5.000 anteriores, desde 1904), el libro de Antonio López de Zuazo resulta de innegable utilidad para el trabajo que estamos proponiendo.

Uno de los últimos intentos sistemáticos que conocemos en España es el libro de Eliseo Izquierdo Periodistas canarios siglos XVIII al XX: propuestas para un diccionario biográfico y de seudónimo, publicado en tres volúmenes en 2005.

Finalmente, otro intento, menos sistemático pero igualmente muy valioso, de llevar a cabo biografías de periodistas es la serie que en 1923 y 1924 publicó el Anuario de la Asociación de la Prensa Diaria de Barcelona, titulada Vida de periodistas ilustres. Se publicaron, en dos tomos, las de Margall, Mañé i Flaquer, Feliu i Rodi, Milà i Fontanals, Joan Maragall, Francisco Peris Mencheta y Luis Figuerola Anglada.

El modelo más claro, y para un colectivo mucho más reducido, lo que es una innegable ventaja en el diseño investigador y en cronograma a adoptar, es el Dictionnaire des Journalistes (1600-1789) del grupo coordinado por Jean Sgard parte de un trabajo previo, publicado por las Presses Universitaires de Grenoble, de 1976. La edición definitiva, de 1999, cuenta con 2000 biografías, las de todos los periodistas de habla francesa de los siglos XVII y XVIII. Cada biografía se expone siguiendo ocho epígrafes: estado civil, formación, carrera profesional, situación económica, opiniones, actividad 
periodística, publicaciones y bibliografía. El prestigioso historiador Roger Chartier ha sugerido, en una elogiosa reseña al Dictionnaire de Sgard, que se amplíe en una versión electrónica, mucho más fácilmente actualizable. No podemos estar más de acuerdo con la propuesta.

La estrategia concreta (sobre cuestiones metodológicas generales, seguimos en buena medida a Urquijo, 2013 y 2014) que planteamos llevar a cabo seguirá los siguientes pasos:

1) Definir el grupo: En principio, los trabajadores de ambos medios de comunicación, con especial atención a aquellos que se dedicaron a la información. El volumen relativamente manejable de personas que en total compusieron aquellas redacciones entre 1998 y 2009, algo más de una década, haría aconsejable al menos censar no sólo a periodistas, fotógrafos o productores de vídeo, sino también a otro tipo de perfiles, técnicos informáticos, por ejemplo. Tengamos en cuenta que, incluso a manera de hipótesis, la polivalencia profesional es un rasgo que en otras investigaciones (por ejemplo, la que se recoge en el volumen colectivo coordinado por López García y Pereira Fariña, 2010) ha aparecido como consustancial a las redacciones de medios digitales, y por tanto no siempre será fácil discernir funciones. En cualquier caso, establecer como categoría de análisis (y como campo de esa base de datos) las funciones profesionales a investigar contribuirá a evitar distorsiones y hacer que el colectivo social a estudiar muestre más características homogéneas que heterogéneas.

2) La incardinación social, incluso en términos de clase social (como hacen, por ejemplo, Friedman, O’Brien, Laurison, 2017), es decir, tipo de familia, formación, ingresos, estudios (formación reglada universitaria, estudios complementarios), y formación en nuevas tecnologías y en destrezas profesionales, trayectoria profesional, pertenencia a agrupaciones de tipo sindical, profesional, cultural o de otro tipo, si se trataba de su primer trabajo como periodista o no, serán aspecto a tener en cuenta. Asimismo, como dato no únicamente numérico nos interesa saber a qué edad se desempeñaron en esos medios, y hasta qué punto hablamos de un carrera profesional, con características propias, ligadas también a la edad.

3) Mediante entrevistas, recabaremos también información sobre lecturas que les hayan influido, así como otro tipo de inputs culturales que hayan podido incidir en la formación del perfil profesional que investigamos (Cook, 2012). Si han tenido contacto con periodistas en el extranjero.

4) También nos interesa, igualmente mediante entrevistas, el tipo de contactos y relaciones tanto con miembros del colectivo estricto que estudiamos como con miembros del colectivo más amplio de informadores, bien de otro tipo de medios del entorno, bien de otros medios digitales, bien extranjeros.

Una prosopografía tiene que realizar las mismas preguntas a las vidas de los biografiados para obtener un producto coherente y homogéneo. Eso se consigue mediante una ficha tipo. Número de registro 
1. Nombre: apellidos y nombre

2. Otra(s) forma(s) del nombre y pseudónimo(s) utilizado(s)

3. Lugar de nacimiento

4. Lugar de defunción (si procede)

5. Fecha de nacimiento

6. Fecha de defunción (si procede)

7. Obras escritas por el personaje (libros)

8. Obras escritas por el personaje (artículos de prensa)

9. Bibliografía sobre el personaje

10. Documentos sobre el personaje

11. Imágenes del personaje

12. Antecedentes familiares

13. Matrimonio

14. Hijos

15. Parientes

16. Domicilio(s) conocido(s) [ciudad o ciudades de residencia en el periodo estudiado]

17. Formación académica

18. Ejercicio profesional como periodista.
a. Empresas.
b. Periodo de contrato.
c. Tipo de contrato.
d. Actividades y funciones que realizó

19. Ejercicio profesional (otras actividades)

20. Participación en empresas

21. Participación en asociaciones

22. Patrimonio: rentas y fiscalidad

23. Premios y homenajes

24. Ideología

25. Militancia política

26. Militancia sindical

27. Cargos administrativos y electos, si los tuviese

28. Lenguas utilizadas

Uno de los campos más importantes es, obviamente, el 10: artículos de prensa escritos por el personaje biografiado. Ello permitiría a su vez confeccionar una base de datos que incluiría el título, publicación donde se publicó el texto (o la fotografía o dibujo: pretendemos considerar periodista a todo informador, independientemente de la técnica y el lenguaje que utilice), fecha de la misma y número de páginas. Esos campos, más otro que recoja el texto completo de ese artículo, noticia, reportaje, entrevista, fotografía, dibujo, caricatura, etc., constituirían la base de datos de trabajos periodísticos. 


\section{Aplicación de otras técnicas: entrevistas semiestructuradas y análisis de redes sociales}

Junto con las técnicas prosopográficas, que serán centrales en nuestro análisis, proponemos aplicar a su vez y de forma coordinada con estas otras dos técnicas de las ciencias sociales: las entrevistas semiestructuradas y el análisis social de redes.

Sobre la entrevista etnográfica, y concretamente la variedad semiestructurada, existe una copiosa bibliografía. Baste citar aquí, a modo de introducción, Hammer y Wildavski, 1999, Kvale y Brinkmann, 2009, King y Horrocks, 2010, y más recientemente y sobre entrevistas a periodistas, concretamente, Tracy y Robles, 2010, y Meyers y Davidson, 2017). La entrevista semiestructurada nos ayudará en dos fases de la investigación: tanto para conocer datos sobre la trayectoria vital y profesional de los entrevistados, de cara a la confección de biografías que alimenten esa prosopografía de un colectivo social, como para conocer sus opiniones, actitudes, creencias, estrategias o modo de adoptar decisiones en el ámbito profesional. Lo que planteamos es realizar, más que historias de vida que se articulan en torno a preguntas muy abiertas que simplemente invitan al entrevistado a hablar, sino interrogantes que buscan unas respuestas muy concretas. Estructuradas, semiestructuradas, focalizadas o abiertas. El cuestionario inicial de la entrevista puede estar fuertemente estructurado, hasta el punto de no admitir cambios ni en las preguntas ni en el orden de las mismas, por lo tanto se trata de un conjunto de preguntas cerradas. Las encuestas, en este caso cuantitativas, son de este tipo. Las entrevistas semiestructuradas, que acostumbran a ser las más utilizadas, plantean una serie de preguntas generalmente organizadas en torno a categorías de análisis, cuyo orden puede cambiar en función del desarrollo de la entrevista. Las focalizadas -casi todas las cualitativas lo son en mayor o menor medida- se centran en obtener información sobre aspectos muy concretos del estudio de investigación que se lleva a cabo. No se trata, al contrario que las historias de vida, de preguntas muy abiertas que simplemente invitan al entrevistado a hablar, sino interrogantes que buscan unas respuestas muy concretas. No obstante, no es descabellado combinar una suerte de historias de vida, que buscan recabar información sobre el individuo, con entrevistas focalizadas, son generalmente temáticas, pero a partir de cuestionarios con un cierto nivel de estructuración, ya que pretendemos obtener un mejor conocimiento de la vida profesional de los individuos del grupo social que estudiamos, y por tanto nos interesa reducir el enfoque de las sesiones. Así, más que entrevistas descriptivas o exploratorias, nos decantamos por un diseño de entrevistas interpretativas, en las que se solicita a los entrevistados que expliquen aspectos concretos de los temas que se les plantean y aporten puntos de vista propios, y de contraste, cuando lo que se busca es 
comparar y comprobar versiones diferentes o confirmar determinados datos previamente obtenidos con otras entrevistas u otras técnicas de investigación.

Las entrevistas semiestructuradas se llevarían a cabo siempre por fases, con posibilidad de volver a entrevistar las mismas personas, tanto para abordar puntos de vista diversos (etnográficos o prosopográficos) como para completar, contrastar o ampliar información. Este método ha sido ya puesto a prueba en el caso de diferentes colectivos profesionales (véase, por ejemplo, Hawe y Gali, 2012, para el caso del colectivo de la enseñanza primaria, o Sabot et al. , 217, para los profesionales de la salud; para el caso de los diarios [digitales], y para aspectos un tanto diferentes, véase por ejemplo Welbers y Opgenhaffen, 2018, y en menor medida, Anderson, 2013), y se ha revelado una herramienta excelente para el análisis de sistema sociales complejos (Quin, Woehle y Tiemann, 2012). La propuesta, para el caso que proponemos como ejemplo y que ya hemos empezado a trabajar, es entrevistar primero a los responsables de los dos medios que investigamos, luego a los trabajadores, y en tercer lugar a otras personas relevantes del panorama del periodismo y la comunicación digital de la época, y tratarlas sistemáticamente mediante utilización de software CAQDAS (Computer Assisted Qualitative Data Analysis), en torno a la producción de categorías sistemáticas. La primera tanda de entrevistas semiestructuradas que llevamos a cabo a lo largo de 2017 con los primeros responsables de aquellas redacciones del Diario de Barcelona digital y de La Malla pusieron de manifiesto aspectos interesantes a desarrollar acerca del papel de instituciones, empresas y medios, de la formación de los periodistas, de la construcción de trayectorias profesionales, del desarrollo de lenguajes informativos propios adaptados a lo digital, de estrategias, actitudes y opiniones profesionales que nos ayudarán en el futuro a plantear una etnografía de esas redacciones digitales (véase, al respecto, y sin ánimo de ser exhaustivos, Willig, 2012; Dickinson, Matthews y Saltzis, 2013; Tamelin y Broersma, 2013, Carson, 2015, Usher, 2015, Robinson y Metzler, 2016, Rosenberg, 2017).

No se descarta, a este respecto, la adopción de otras técnicas de investigación, como los paneles de expertos (presenciales, de manera que los participantes se sometan a un mismo estatus) o el método Delphi (no presencial, para aquellas situaciones en que unos miembros del grupo puedan influir en otros) para recabar información de especialistas.

La otra gran técnica a aplicar sería el análisis de redes sociales (social network analysis, SNA). Se basa, a partir de la teoría de grafos (se emplean normalmente programas que combinan las matrices con la producción de grafos, como NodeXL), en determinar las relaciones en una red social entre nodos, en este caso personas que trabajaron para dos medios que constituyen casos paradigmáticos, y explicar mediante qué canales y modos de difusión se produce esa relación entre nodos o actores, o, en nuestro caso, de qué manera circula y se comparte la información en una red dada. El objetivo último es explicar los procesos en red dentro de movimientos sociales y acciones 
colectivas, en nuestro caso comunicativas. La teoría de grafos nos ofrece una representación de una red como modelo, puesto que el análisis de redes sociales se centra en la observación de las relaciones de los actores en entornos contextualizados (Bellotti, 2015: 15), como un modo de concebir los sistemas sociales determinados, en nuestra propuesta una emergente práctica profesional, la periodística, adaptada al entorno digital en un contexto determinado, como la Barcelona post-olímpica, prestando atención a las relaciones entre diferentes entidades (personas $u$ otras, por ejemplo organizaciones de medios o instituciones públicas en una red de distribución de noticias) que componen el sistema. Por decirlo de otra manera, las redes sociales son un fenómeno empírico que presenta una relación entre actores mediante patrones de relaciones interconectadas. Describir, visualizar y explicar esos patrones es el objetivo principal de la ciencia y el análisis de redes, mediante parámetros que afectan a la red en su conjunto, como la densidad, o relación entre el número de relaciones posibles y el número de relaciones de las que tenemos constancia, la transitividad, o posibilidad de que dos nodos estén conectados entre sí cuando ambos están conectados a un tercer nodo, y la reciprocidad, cuando las relacionales son bilaterales, y parámetros que se refieren a las propiedades de cada nodo, en nuestro caso cada persona biografiada en el análisis prosopográfico, y que se basan en los grados y tipos de centralidad de cada nodo. Estos son el grado de centralidad propiamente dicho, o número de conexiones que cada nodo recibe de otros; el grado de interinidad, o número de veces que un nodo actúa como puente o intermediario en el camino más corto posible entre otros dos nodos, o potencial de un nodo de controlar flujos de información en una red, el grado de cercanía, o por cuántas relaciones debe pasar un nodo antes de llegar a otro nodo, y el eigenvector, o estatus de un nodo, determinado tanto por el número de conexiones directa que recibe como de lo bien conectados que sus contactos están a su vez. Esto quiere decir que un nodo con una interinidad alta que muestra una influencia notable en la transferencia de información en una red, que una interinidad alta muestra una influencia notable en la transferencia de información en una red, ya que la información siempre sigue el camino más corto, y muestra también un potencial de un nodo de controlar flujos de información en una red. Un análisis de redes (que debería incluir también como nodos a las fuentes informativas mencionadas en la producción periodistica en los dos medios analizados como ejemplo por los periodistas biografiados) nos permitiría cómo es flujo informativo de la red que estudiamos, por qué caminos llega la información a su destino y cuàntos pasos da.

\section{A modo de recapitulación}

En definitiva, lo que proponemos en estas pocas páginas es sentar las bases metodológicas para un estudio que se centre básicamente en la reconstrucción de las trayectorias profesionales de un colectivo determinado, en este caso los primeros 
periodistas digitales de Barcelona, a través de la reconstrucción de la historia de dos medios de iniciativa pública durante una década larga (1998-2009), el Diario de Barcelona digital y La Malla, del que ya hemos presentado algunos resultados provisionales (Díaz Noci, 2018, en prensa). Se trata así, de un work in progress que pretende beneficiarse de una triangulación metodológica que comprende métodos de la historiografía y de las ciencias sociales, en concreto métodos cualitativos, con el objetivo de realizar una aportación al campo de la etnografía de las redacciones. Queremos que el método se base en la realización de un censo de directivos y trabajadores de esos medios, en el establecimiento de unas categorías de análisis biográficas sistemáticas, y que pueda dar lugar como resultados de investigación a un diccionario de periodistas digitales, pero también a una explicación interpretativa del fenómeno más profunda, y por tanto más cercana a una perspectiva social. Se trataría de una contribución a explicar en tantas dimensiones como sea posible un momento de transición en el ecosistema mediático, pero también a incorporar técnicas bien conocidas y que han arrojado resultados notables en otros objetos de estudio y para otros colectivos sociales. Una historia si se quiere social, pues tiene en cuenta también las relaciones sociales y laborales (Cohen, 2015; Randle et al., 2015) de los miembros de esos colectivos. Un estudio, en definitiva, de la profesión de periodista (Popkin, 1990 o Cuxac, 2015, para otro momento histórico, y para época más actual, Fontenelle e Silva y Gastal Grill, 2014, Davidson y Meyers, 2016), y en este caso, digital, como ejemplo de la capacidad de transformación (también de resiliencia) de estructuras sociales que se toman como punto de partida.

En cuanto a la presentación de resultados, es importante insistir en la necesidad de confeccionar bases de datos para explotar el material. La información recogida, y clasificada según los apartados de que se compondrá cada biografía, se almacenaría en unas bases de datos coordinadas entre ellas, lo que permitiría la búsqueda de información documental con facilidad, ya que la masa de información que se generaría alcanzaría una dimensión considerable. De hecho, el objetivo técnico del proyecto sería el diseño de un sistema documental apropiado. Los objetivos serían los siguientes:

-Conocer las cabeceras analizadas y acceder a sus datos.

-La consulta de la ficha de cada uno de los periodistas biografiados.

-La consulta de las obras de cada uno de los periodistas cuya obra completa periodística se recoja.

A partir de estas dos primeras bases de datos se llevaría a cabo la explotación sectorial por materias específicas para la elaboración posterior de diferentes estudios.

Como en otras bases de datos de proyectos ligados a la producción de diccionarios biográficos, los campos de esta base de datos normalizada deberían realizar la descripción bibliográfica normalizada (autor/es, título, etc.), a partir del cual, posteriormente, se puede llevar a cabo un análisis del contenido de las obras de cada periodista, periodo, medio de comunicación, zona geográfica, etc., para lo cual se 
establecen los campos referentes a descripción de materias, identificadores personales y corporativos y cronología. Cuando sea posible, se hará en función de tesauros o vocabularios especializados normalizados. Finalmente, la base de datos debe indicar en qué archivo(s) o centro(s) de información se encuentra depositado físicamente el documento. Esto puede ser, como analizamos en el apartado dedicado a los derechos de autor (propiedad intelectual) y de reproducción (propiedad material) del documento en cuestión.

En todo momento se seguirá, para la confección de las bases de datos, la Norrma Internacional General de Descripción Archivística ISAD (G).

Los diccionarios, a medida que se confeccionen, pueden publicarse en forma de libro o bien en forma de página web. A medida que se cierre la transcripción de la obra periodística completa de determinados autores, ésta puede ser publicada en su integridad, en forma de base de datos buscable, en páginas web creadas al efecto.

\section{Referencias bibliográficas}

ANDERSON, C. (2013): Rebuilding the news: Metropolitan journalism in the digital age. Philadelphia, Temple University.

ASENSIO, E.; CALVO, L.; DOMINGO, D. Y RUBIES, T. (2003): Informe sobre la situació laboral i professional del periodista digital a Catalunya, Barcelona, Grup de Periodistes Digitals.

BELLOTTI, E. (2015): Qualitative Networks. Mixed methods in sociological research, New York, Routledge.

BROERSMA, M. (2011): "From press history to the history of journalism" en M\&z, 3, pp. 17-28.

BRÜGGER, N. (2010): Web History, New York, Peter Lang.

BRÜGGER, N. (2012): "Web historiography and Internet Studies: Challenges and perspectives" en New Media \& Society,15(5), pp. 752-764.

BRÜGGER, N. y KOLSTRUP, S. (2002): Media history, Theories, methods, analysis, Aarhus, Aarhus University Press.

BUNOUT, E. (2016): "Visualisation of the prosopography of Polish and German experts on Eastern Europe: Are non-computed data useable for visualisation?" en $D H$ Benelux conference 2016. Disponible en Internet (12-09-2018): http://www.dhbenelux.org/wpcontent/ uploads/2016/05/63BunoutEstelleFinalAbstractDHBenelux2016long.pdf.

CALVO BERMEJO, L. (2005): “El Informe sobre la Situación Laboral y Profesional del Periodista Digital en Cataluña", en Mediatika, 11, pp. 171-183.

COHEN, N. S. (2015): "From Pink Slips to Pink Slime: Transforming Media Labor in a Digital Age" en The Communication Review, 18, pp. 98-122. 
COHEN, L. y MALLON, M. (2001): "My brilliant career? Using stories as a methodological tool in careers research", en. International Studies of Management \& Organization, 31(3), pp. 48-68.

COOK, J. W. (2012): "The kids are all right: On the "turning" of cultural history". en American Historical Review, 117(3), pp. 746-771.

CORELL DOMENECH, M. V. (2013): Científicos, vulgarizadores y periodistas: estudio y análisis de la divulgación de la ciencia en La Ilustración Española y Americana (1869-1898), Tesis doctoral, Valencia, Universidad de Valencia.

CRUZ, A. de la y LÓPEZ-CORDÓN, M. V. (2016): "Impresores que escriben, periodistas que editan en tiempos de crisis y revolución (España 1780-1823)" en El Argonauta Español, 13.

CUXAC, M. (2015): Journaux et journalistes au temps du fascisme. Turin, 1929-1940, Tesis doctoral, Lyon; Torino, Université Lumière Lyon 2; Università degli studi di Torino.

DAVIDSON, R. y MEYERS, O. (2016): "Toward a Typology of Journalism Careers: Conceptualizing Israeli Journalists' Occupational Trajectories" en Communication, Culture \& Critique, 9, pp.193-211.

DÍAZ NOCl, J. (2018, en prensa): “Naixement i consolidació del periodisme a Internet: el cas dels mitjans públics Diari de Barcelona i La Malla en Barcelona. Quaderns d'Història, 24.

DÍAZ NOCI, Javier (2013): "A history of journalism on the Internet: A state of the art and some methodological trends" en Revista internacional de Historia de la Comunicación, 1(1), pp. 253-272.

DICKINSON, R.; MATTHEWS, J. y SALTZINS, K. (2013): "Studying journalists in changing times: Understanding news work as socially situated practiced" en The International Communication Gazette, 75(1), pp. 3-18.

EPSTEIN, E. J. (1973): News from nowhere, London, Random House.

FONTENELLE E SILVA, M. y GASTAL GRILL, I. (2014): “Atuaçao política e produçao escrita de jornalistas no parlamento brasileiro", en Cad. Pes., São Luís, v. 21, n. especial.

FRIEDMAN, S. (2017): “'Like Skydiving without a Parachute': How Class Origin Shapes Occupational Trajectories in British Acting", en Sociology 51(5), pp. 992-1010.

GANS, H. J. (1979): Deciding what's news. A study of CBS Evening News, NBC Nightly News, Newsweek and Time, Chicago, Northwestern University Press.

HAWE, P. y GHALI, L. (2007): "Use of social network analysis to map the social relationshipsof staff and teachers at school", Health Education Research, 23(1), pp. 62-69.

JULIÁ, S. (1989): Historia social, sociología histórica, Madrid, Siglo XXI. 
KEATS-ROHAN, K. S. B. (ed.) (2007): Prosopography Approaches and Applications: A Handbook, Oxford, University of Oxford Linacre College Unit for Prosopographical Research.

KING, N. y HORROCKS, C. (2010): Interviews in Qualitative Research. Thousand Oaks, CA: SAGE Publications.

KVALE, S. y BRINKMANN, S. (2009): Interviews: learning the craft of qualitative research interviewing, Los Angeles, Sage.

LÓPEZ GARCÍA, X. y PEREIRA FARIÑA, X. (2010): Convergencia digital. Reconfiguración de los medios de comunicación en España, Santiago de Compostela, Universidade de Santiago de Compostela.

LULL, J. (1988): World families watch television, London etc.: Sage.

- (1990): Inside family viewing. Ethnographic research on television's audiences, London etc., Sage.

MEYEN, M. y FIEDLER, A. (2013): "Journalists in the German Democratic Republic (GDR): A collective biography" en Journalism Studies, 14(3), pp. 321-335.

MEYEN, M. y WIEDEMANN, T. (2017): "Journalism Professors in the German Democratic Republic: A Collective Biography" en International Journal of Communication 11(2017), pp. 1839-1856.

MEYERS, O. y DAVIDSON, R. (2015): "Toward a Typology of Journalism Careers: Conceptualizing Israeli Journalists' Occupational Trajectories" en Communication, Culture \& Critique 9, pp. 193-211

MEYERS, O. y DAVIDSON, R. (2017): "Interviewing interviewers: Collecting, analyzing and generalizing from occupational life histories of journalists" en The Communication Review, 20(4), pp. 277-295.

OLDFIELD, S. J. (2015): "Narrative Methods in Sport History Research: Biography, Collective Biography, and Prosopography" en International Journal of the History of Sport, 32(15) pp. 1855-188

OLDFIELD, S. J. y DAY, D. (2011): Narrative, Biography and Prosopography: Historical Method and its Implications. MMU Institute for Performance Research, Sports Coaching: Pasts and Futures Conference, Saturday 25 - Sunday 26 June at Wychwood Park, Cheshire.

ONANDIA MARTÍNEZ, A. (2018): De la biografía a la prosopografía: Los parlamentarios de la Comunidad Autónoma Vasca (1977-1982), Vitoria-Gasteiz, Parlamento Vasco.

PATERSON, C. y DOMINGO, D. (2008): Making online news: The ethnography of new media. New York: Peter Lang.

PONT-SORRIBES, C.; LUIS, R..; SANMARTÍ, José M. (2017): “Aproximación etnográfica del periodista de la Transición como fuente histórica: Estudio de la relación con los actores políticos y de los cambios en la producción de los medios escritos" en Historia y Comunicación Social, 22(1), pp. 141-156.

POOLEY, J. D. y PARK, D. W. (2013): “Communication research”. En Simonson, P. et al., 
The handbook of communication history, London, Routledge, pp. 76-90.

POPKIN, J. D. (1990): “The Press and the French Revolution after Two Hundred Years" en French Historical Studies, 16(3), pp. 664-683.

QUINN, A.; WOEHLE, R. y TIEMANN, K. (2012): "Social network analysis for analyzing groups as complex systems", Journal of Social Service Research, 38(5), pp. 605618.

RANDLE, K. et al. (2015): "Towards a Bourdieusian analysis of the social composition of the UK film and television workforce" en Work, employment and society, 29(4), pp. 590-606.

RAYMOND, J. (1999): "The History of Newspapers and the History of Journalism: Two Disciplines or One?" en Media History, 5(2), pp. 223-232.

RODRIGO ALSINA, M. (1989), La construcción de la noticia, Barcelona, Paidós.

ROSENBERG, L. (2017): “Etnografía del trabajo periodístico. Apuntes sobre la inserción del investigador en la sala de redacción de un diario argentino" en Comunicación y sociedad, 28, pp. 87-109.

SABOT, K., et al. (2017): "Use of social network analysis methods to study professional advice and performance among healthcare providers: a systematic review" en Systematic Reviews, 6, pp. 208 ss.

SAMUEL, R. (1985): "What is Social History?" History Today, 35(3).

SAVILLE-TROIKE, M. (1989): The Ethnography of Communication: An Introduction, Oxford, Basil Blackwell.

SCHUDSON, M. (1981): Discovering the news: A social history of American newspapers, New York, Basic Books.

- (1990): The sociology of news, New York, W: W. Norton \& Co.

SCOTT, Ben (2005): "A contemporary history of digital journalism" en Television \& New Media, 6(1), pp. 89-126.

SIMONSON, P. y PARK, D. W. (2015): The international history of communication study, London, Routledge.

SORIANO CLEMENTE, J. (2017): “Investigar la comunicación con métodos biográficos. Propuestas de estudio" en Historia y Comunicación Social, 22(1), pp. 157-171.

TAMELING, K. y BROERSMA, M. (2013): "De-converging the newsroom: Strategies for newsroom change and their influence on journalism practice" en The International Communication Gazette, 75(1), pp. 19-34.

TRACY, K. y ROBLES, J. S. (2010): “Challenges of Interviewers' Institutional Positionings: Taking Account of Interview Content and the Interaction" en Communication Methods and Measures, 4(3), pp. 177-200,

TSUI, C. Y. S. y LEE, F. L. F. (2012): "Trajectories of women's journalists careers in Hong Kong. A field theory perspective" en Journalism Studies, 13(3), pp. 370-385. 
TUCHMAN, G. (1978): Making news. A study in the construction of reality, New York, The Free Press.

URQUIJO, M. (2013): “Renovación metodológica de los diccionarios biográficos nacionales", Erebea. Revista de Humanidades y Ciencias Sociales, 3, pp. 249-270.

- (2014): "Prosopografía parlamentaria a partir de la biografía colectiva: algunas reflexiones metodológicas", en Agirreazkuenaga, J. y Alonso, E. J. (eds.), Naciones en el Estado-nación: La formación cultural y política de naciones en la Europa contemporánea. Barcelona, Base, pp. 57-63.

USHER, N. (2014): Making news at The New York Times, Ann Arbor, University of Michigan Press.

- (2015): "Newsroom moves and the newspaper crisis evaluated: Space, place, and cultural meaning" en Media, Culture \& Society, 37(7), pp. 1005-1021.

WELBERS, K. y OPGENHAFFEN, M. (2018): "Social media gatekeeping: An analysis of the gatekeeping influence of newspapers' public Facebook pages", New Media \& Society DOI https://doi.org/10.1177/1461444818784302

WELLER, T. (ed.) (2013): History in the Digital Age, London; New York, Routledge.

WILLIG, I. (2012): "Newsroom ethnography in a field perspective" en Journalism, 14(3), pp. 372-387. 